\title{
A PROOF OF SOME IDENTITIES OF RAMANUJAN USING MODULAR FORMS
}

\author{
by ANTHONY J. F. BIAGIOLI
}

(Received 18 May, 1988)

Introduction. In $1974 \mathrm{~B}$. J. Birch [1] published a description of some manuscripts of Ramanujan which contained, among other things, a list of forty identities involving the Rogers-Ramanujan functions

and

$$
G(x)=\prod_{n=0}^{\infty} \frac{1}{\left(1-x^{5 n+1}\right)\left(1-x^{5 n+4}\right)},
$$

$$
H(x)=\prod_{n=0}^{\infty} \frac{1}{\left(1-x^{5 n+2}\right)\left(1-x^{5 n+3}\right)} .
$$

At that time nine of these had been proven, and since then twenty-two more of them have been proven, fifteen of them by David Bressoud in his thesis [2]. Bressoud gives a synopsis of the extant proofs, where he attributes proofs to H. B. C. Darling [3], L. J. Rogers [4], L. J. Mordell [5], and G. N. Watson [6].

$G(x)$ and $H(x)$ can be expressed as theta functions by using the Jacobi triple product identity. Substituting $x=e^{2 \pi i \tau}$ we find that $g(\tau)=x^{-1 / 60} G(x)$ and $h(\tau)=x^{11 / 60} H(x)$ are modular forms of weight 0 on a certain subgroup $\Gamma_{0,1}(5,5) \leq \Gamma(1)$ of the modular group, and for any positive integer $n$, the functions $g(n \tau)$ and $h(n \tau)$ will be modular forms on an appropriate subgroup. It requires some work to get the multiplier system for these functions in a workable form, but this is done in $\$ 2$.

The identities involve the functions $G(x)$ and $H(x)$ only in the combinations

$$
U_{r, s}= \begin{cases}G\left(x^{r}\right) G\left(x^{s}\right)+x^{(r+s) / 5} H\left(x^{r}\right) H\left(x^{s}\right), & \text { when } r+s \equiv 0(\bmod 5), \\ G\left(x^{r}\right) H\left(x^{s}\right)-x^{(r-s) / 5} H\left(x^{r}\right) G\left(x^{s}\right), & \text { when } r-s \equiv 0(\bmod 5) .\end{cases}
$$

In addition, the functions

and

$$
P(x)=\prod_{n=1}^{\infty}\left(1-x^{2 n-1}\right)
$$

$$
P^{*}(x)=\prod_{n=1}^{\infty}\left(1+x^{2 n-1}\right)
$$

appear. With these notations, and preserving Birch's numeration, eight of the identities are:

$$
\begin{gathered}
U_{6,14}=U_{42,2} \\
U_{6,14}=\frac{1}{2 x}\left[P^{*}(x) P\left(x^{3}\right) P^{*}\left(x^{7}\right) P\left(x^{21}\right)-P(x) P^{*}\left(x^{3}\right) P\left(x^{7}\right) P^{*}\left(x^{21}\right)\right] \\
U_{2,13}^{2}=\frac{P\left(x^{13}\right)-x P(x)}{P\left(x^{13}\right) P(x)}
\end{gathered}
$$

Glasgow Math. J. 31 (1989) 271-295. 


$$
\begin{gathered}
{\left[U_{17,2} U_{1,34}\right]^{4}-x\left[U_{17,2} U_{1,34}\right]^{2}=\frac{P\left(x^{17}\right)^{3}-x^{2} P(x)^{3}}{\left[P\left(x^{17}\right) P(x)\right]^{3}}} \\
U_{2,23} U_{46,1}=P(x) P\left(x^{23}\right)+x+\frac{2 x^{2}}{P(x) P\left(x^{23}\right)} \\
P\left(x^{11}\right) U_{2,33}=P\left(x^{3}\right) U_{66,1} \\
P(x) U_{3,22}=P\left(x^{33}\right) U_{11,6} \\
{\left[U_{1,94} U_{47,2}-P(x) P\left(x^{47}\right)-2 x^{2}-\frac{2 x^{4}}{P(x) P\left(x^{47}\right)}\right]^{2}=4 x^{2} P(x) P\left(x^{47}\right)+9 x^{4}+\frac{8 x^{6}}{P(x) P\left(x^{47}\right)}}
\end{gathered}
$$

$P(x)$ and $P^{*}(x)$ can be expressed in terms of the Dedekind eta function, and the functions $U_{r, s}$ yield modular forms when multipled by the appropriate power of $x$,

$$
u_{r, s}(\tau)= \begin{cases}g(r \tau) g(s \tau)+h(r \tau) h(s \tau), & \text { for } r+s \equiv 0(\bmod 5), \\ g(r \tau) h(s \tau)-g(s \tau) h(r \tau), & \text { for } r-s \equiv 0(\bmod 5),\end{cases}
$$

which are forms of weight 0 on the subgroup which is the intersection of the subgroups for $g(r \tau), g(s \tau), h(r \tau)$, and $h(s \tau)$.

To get a proof of any one of these identities from this point requires knowing a complete set of inequivalent cusps for the subgroup, checking that the multiplier systems of each term in the identity agree, having a lower bound for the order of the identity at each cusp, and then calculating sufficiently many of the coefficients of $x^{n}$ in the series expansion to see that the valence formula ( $\mathrm{cf}(1.5)$ ) would be violated if the function were not identically zero. This is a task which can certainly be done, with a computer, but without further simplifications it is necessary to compute several hundred terms in the series expansion.

To reduce the complexity of the computation we can try looking closer at the modular properties of the functions $u_{r, s}$. More turns out to be true than we have a right to expect.

First of all we shall show that $u_{r, s}$ is a modular form on the subgroup $\Gamma_{0}([r, s])$, whose index is 12 times smaller than that of the subgroup for $g(r \tau)$ and $g(s \tau)$. This translates to a reduction by a factor of more than 12 in the number of terms necessary to be computed.

Besides this, there is an obvious symmetry in the above identities, when there is more than one prime factor involved, as we can see most clearly by comparing (\#36) and (\#37). We make this symmetry explicit by applying what we call the Fricke involutions, whose properties are developed in $\S 4$. After doing this, it turns out that for a proof of some of the indentities it is only necessary to check that the constant term vanishes, while only one requires us to do as many as 5 terms.

Thus, we have managed to replace a lot of computation with a fair amount of theory, which seems good in that it leads us to a better understanding of these functions. However, this technique is only a technique of verification: it does not show us how to construct new identities, only how to tell whether one we already have in hand is true or not. 
After completing the proofs of the identities which involve $u_{r, s}$ there is one identity which remains unproven. It is

$$
P\left(x^{38}\right) U_{19,4}=P\left(x^{2}\right) U_{76,1}^{*},
$$

where, with $G^{*}(x)=G(-x)$, and $H^{*}(x)=H(-x)$,

$$
U_{76,1}^{*}=G\left(x^{76}\right) H^{*}(x)+x^{15} G^{*}(x) H\left(x^{76}\right) .
$$

This identity is not quite in the same mold as the other eight we consider, but a slight adaption of the techniques used on them does yield a proof.

1. Background on modular forms. For a general theory of modular forms we refer the reader to Rankin [7], Schoeneberg [8], Knopp [9], and Rademacher [10], although the notation we use here is not strictly in accordance with any one of these.

By $M_{2}^{+}(\mathbf{R})$ and $M_{2}^{+}(\mathbf{Z})$ we denote the sets of $2 \times 2$ matrices with positive determinant and real and integral entries, respectively. The modular groups is

$$
\Gamma(1)=\left\{A \in M_{2}^{+}(\mathbf{Z}) \mid \operatorname{det}(A)=1\right\},
$$

the principal transform subgroup of level $n$ is

$$
\Gamma_{0}(n)=\left\{A=\left(\begin{array}{ll}
a & b \\
c & d
\end{array}\right) \in \Gamma(1) \mid c \equiv 0(\bmod n)\right\} .
$$

For $M=\left(\begin{array}{ll}a & b \\ c & d\end{array}\right) \in M_{2}^{+}(\mathbf{R})$ we define the bilinear transformation

$$
M \tau=\frac{a \tau+b}{c \tau+d},
$$

for any $\tau \in \mathbf{H}=\{\tau \in \mathbf{C}: \operatorname{Im} \tau>0\}$, and the automorphic factor

$$
(M: \tau)=c \tau+d .
$$

For any $f: \mathbf{H} \rightarrow \mathbf{C} \cup\{\infty\}$ the stroke operator of weight $r$ is the operator $f \mapsto f \mid M$ defined by

$$
f \mid M=(\operatorname{det} M)^{+r / 2}(M: \tau)^{-1} f(M \tau) .
$$

When $r=0$ this is $f \mid M(\tau)=f(M \tau)$, and when $M=\left(\begin{array}{cc}m & 0 \\ 0 & n\end{array}\right)$ the bilinear transformation is $M \tau=\frac{m}{n} \tau$. We write $f \mid m$ and $f \mid \frac{m}{n}$ as abbreviations for $f \mid\left(\begin{array}{cc}m & 0 \\ 0 & 1\end{array}\right)$ and $f \mid\left(\begin{array}{cc}m & 0 \\ 0 & n\end{array}\right)$. Although $f|M| A=f \mid M A$ does not hold in general, we do have this equality when $r$ is an integer; or when $M$ or $A$ is of the form $\left(\begin{array}{ll}a & b \\ 0 & d\end{array}\right)$ with both $a$ and $d$ positive. For $A=\left(\begin{array}{ll}a & b \\ c & d\end{array}\right)$ and $n$ a natural number, we define two auxiliary matrices ${ }^{n} A$ and ${ }_{n} A$ so as 
to satisfy

$$
f|n| A=\left.f\right|^{n} A|n=f|{ }_{n} A \mid \frac{1}{n}
$$

or, in other words,

$$
{ }^{n} A=\left(\begin{array}{cc}
a & b n \\
c / n & d
\end{array}\right) \quad \text { and } \quad{ }_{n} A=\left(\begin{array}{cc}
n a & b \\
c & d / n
\end{array}\right) .
$$

In addition, we shall use ${ }_{n}^{m} A={ }^{m}\left({ }_{n} A\right)$.

We say $f$ is a modular form if it is a form on some $\Gamma$ of finite index in $\Gamma(1)$. The functional property which a modular form must satisfy is its functional relation:

$$
\left.f\right|_{(r)} A=v(A), f \text { for all } A \in \Gamma \text {. }
$$

$v(A)$ is called the multiplier system of $f$ and $r$ is its weight.

If $f$ is a modular form on $\Gamma$ and $n$ is a natural number, then $f \mid n$ is a modular form of the same weight on the group $\Gamma^{\prime}=\left\{A \in \Gamma(1) \mid{ }^{n} A \in \Gamma\right\}$ with multiplier system $v \mid n$ defined by

$$
v \mid n(A)=v\left({ }^{n} A\right) \text {. }
$$

If $f$ and $g$ are two modular forms on $\Gamma$ and $\Gamma^{\prime}$, respectively, then $f . g$ is always a modular form on $\Gamma \cap \Gamma^{\prime}$. However, $f+g$ is only a modular form when their weights are equal, and then it is a modular form on the subgroup

$$
\Gamma^{\prime \prime}=\left\{A \in \Gamma \cap \Gamma^{\prime} \mid v(A)=v^{\prime}(A)\right\},
$$

provided this has finite index in $\Gamma(1)$. The first step in each of our proofs will be to find the subgroup on which all of the multiplier systems agree.

Any non-zero modular form of weight 0 must satisfy the valence formula:

$$
\sum_{z \in \mathscr{F} *} \operatorname{Ord}_{\Gamma}(F ; z)=0
$$

where $\mathscr{F}^{*}$ is a fundamental set for $\Gamma$. We shall use this to prove that a modular form is identically zero by showing that it fails.

We shall need the following properties of $\Gamma_{0}(n)$.

LEMMA 1.1. If $(r, s)=1$, then the width of $\Gamma_{0}(n)$ at $\frac{r}{s}$ is

$$
\kappa\left(\Gamma_{0}(n) ; \frac{r}{s}\right)=\frac{n}{\left(n, s^{2}\right)}
$$

Proof. If $B \in \Gamma(1)$ is such that $\frac{r}{s}=B^{-1}(\infty)$, then

$$
B^{-1} U^{k} B=\left(\begin{array}{cc}
1-k r s & k r^{2} \\
-k s^{2} & 1+k r s
\end{array}\right) \in \Gamma_{0}(n)
$$

if and only if $n$ divides $k s^{2}$. 
LEMMA 1.2. If no square divides $n$ other than 1 or 4 , then a complete set of inequivalent cusps for $\Gamma_{0}(n)$ is $\left\{\frac{1}{a}: a \mid n\right\}$.

LEMMA 1.3. If no square divides $n$ other that 1 or 4 , and if $(r, s)=\left(r^{\prime}, s^{\prime}\right)=1$, then $\frac{r}{s}$ and $\frac{r^{\prime}}{s^{\prime}}$ are equivalent cusps modulo $\Gamma_{0}(n)$ if and only if $(s, n)=\left(s^{\prime}, n\right)$. (We allow $\infty$ in the form $\infty=\frac{1}{0}$.)

Lemmas 1.2 and 1.3 are proven in Berndt, Biagioli, and Purtilo [12], [13].

If $\operatorname{Ord}_{\Gamma}(f ; \zeta)$ denotes the order of $f$ with respect to $\Gamma$ at the cusp $\zeta \in \mathbf{Q} \cup\{\infty\}$ and $\kappa(\Gamma ; \zeta)$ denotes the width of $\Gamma$ at $\zeta$, then the invariant order, $\operatorname{ord}(f ; \zeta)$ is defined by the equation

$$
\operatorname{Ord}_{\Gamma}(f ; \zeta)=\kappa(\Gamma ; \zeta) \operatorname{ord}(f ; \zeta)
$$

It has the following property for the order of the transform $f \mid M$ of $f$ by the matrix $M=$ $\left(\begin{array}{ll}a & b \\ c & d\end{array}\right) \in M_{2}^{+}(\mathbf{Z})$ : If $r, s \in \mathbf{Q},(r, s)=1, m=\operatorname{det}(M)$, and if $g=(a r+b s, c r+d s)$ (this is the factor which cancels from the numerator and denominator when $M_{s}^{r}=\frac{a r+b s}{c r+d s}$ is
reduced to lowest terms), then

$$
\operatorname{ord}\left(f \mid M ; \frac{r}{s}\right)=\frac{g^{2}}{m} \operatorname{ord}\left(f ; M \frac{r}{s}\right) .
$$

(See Berndt, Biagioli and Purtilo [11].)

Suppose $d \| n$, i.e., $d \mid n$ and $\left(d, \frac{n}{d}\right)=1$. Then a Fricke involution of level $d$ for $\Gamma_{0}(n)$ is a transformation $M=B\left(\begin{array}{ll}n & 0 \\ 0 & 1\end{array}\right)$, where $B=\left(\begin{array}{ll}\alpha & \beta \\ \gamma & \delta\end{array}\right) \in \Gamma_{0}\left(\frac{n}{d}\right)$ and $\delta \equiv 0(\bmod d)$. These satisfy the following property:

LEMMA 1.4. If $d \| n, f$ is a form on $\Gamma=\Gamma_{0}(n)$, and $M$ is a Fricke involution of level $d$ for $\Gamma_{0}(n)$, then for every $z \in \mathbf{H} \cup \mathbf{Q} \cup\{\infty\}$,

$$
\operatorname{Ord}_{\Gamma}(f \mid M ; z)=\operatorname{Ord}_{\Gamma}(f ; M z) .
$$

Proof. For $z \in \mathbf{Q} \cup\{\infty\}$ this was proved partially in [13], and the complete proof is similar to the one given there. For a point $z \in \mathbf{H}$, this follows because $P \in \Gamma_{0}(n)$ if and only if $M P M^{-1} \in \Gamma_{0}(n)$, so that $z$ and $M z$ are both non-fixed points of $\Gamma_{0}(n)$ or they are both fixed points of the same order, and $f \mid M$, as an analytic function in $\mathbf{H}$ has the same order at $z$ as $f$ does at $M z$.

LEMmA 1.5. Suppose $d \| n, m \mid n, B=\left(\begin{array}{ll}\alpha & \beta \\ \gamma & \delta\end{array}\right)$, and $M=B\left(\begin{array}{ll}d & 0 \\ 0 & 1\end{array}\right)$ is a Fricke involution of level $d$ for $\Gamma_{0}(n)$, and suppose $f$ is a form on $\Gamma_{0}\left(\frac{n}{m}\right)$ with multiplier system $v$. 
Setting $v=(d, m), \mu=\frac{m}{v}, m^{\prime}=\frac{d m}{(d, m)^{2}}$, and $B^{\prime}={ }_{v}^{\mu} B=\left(\begin{array}{cc}v \alpha & \mu \beta \\ \gamma / \mu & \delta / v\end{array}\right), \quad$ this agrees with the notation introduced in (1.3)), then

$$
f|m| M=v\left(B^{\prime}\right) \cdot f \mid m^{\prime}
$$

This says, essentially, that, for a Fricke involution, the stroke operator changes $f \mid m$ into $f \mid m^{\prime}$. The proof follows from

$$
f|m| M=\left.f|\mu v| B|d=f| \mu\right|_{v} B\left|\frac{d}{v}=f\right|{ }_{v}^{\mu} B \mid \mu \frac{d}{v},
$$

since $m^{\prime}=\mu \frac{d}{v}$ and ${ }_{v}^{\mu} B=\left(\begin{array}{cc}v \alpha & \mu \beta \\ \gamma / \beta & \delta / v\end{array}\right) \in \Gamma_{0}\left(\frac{n}{m}\right)$.

LeMMA 1.6. Suppose $d \| n, a \mid n, n$ is divisible by no square other than 1 or 4 , and $M$ is a Fricke involution of level $d$ for $\Gamma_{0}(n)$. Then with $a^{\prime}=\frac{d a}{(d, a)^{2}}$, the cusp $M\left(\frac{1}{a}\right)$ is equivalent modulo $\Gamma_{0}(n)$ to $\frac{1}{a^{\prime}}$.

Proof. Setting $M=B\left(\begin{array}{ll}d & 0 \\ 0 & 1\end{array}\right)$ with $B=\left(\begin{array}{ll}\alpha & \beta \\ \gamma & \delta\end{array}\right) \in \Gamma_{0}\left(\frac{n}{d}\right)$ and $\delta \equiv 0(\bmod d)$, and setting $d_{0}=\frac{d}{(a, d)}, a_{0}=\frac{a}{(a, d)}$, we have

$$
M\left(\frac{1}{a}\right)=B\left(\frac{d_{0}}{a_{0}}\right)=\frac{\alpha d_{0}+\beta a_{0}}{\gamma d_{0}+\delta a_{0}}
$$

Since $\operatorname{det}(B)=1$, this fraction is reduced, and we can apply Lemma 1.3. Using $d\left|\delta, \frac{m}{d}\right| \gamma,\left(\frac{n}{d}, \delta\right)=(d, \gamma)=1$, and $\left(d, \frac{n}{d}\right)=1$, we have

$$
\left(\gamma d_{0}+\delta a_{0}, n\right)=\left(\gamma d_{0}+\delta a_{0}, d\right)\left(\gamma d_{0}+\delta a_{0}, \frac{n}{d}\right)=d_{0} \cdot a_{0}=a^{\prime}
$$

and we are done.

2. Transformation properties and multiplier systems. We begin with some properties of the Dedekind eta function

and the theta function

$$
\eta(\tau)=e^{2 \pi i \tau / 24} \prod_{n=1}^{\infty}\left(1-e^{2 \pi i n \tau}\right)
$$

$$
\vartheta_{1}(v \mid \tau)=-i \sum_{m=-\infty}^{\infty}(-1)^{m} e^{\pi i \tau(m+1 / 2)^{2}} e^{2 \pi i v(m+1 / 2)}
$$


which are defined for $\tau \in \mathbf{H}$ and $v \in \mathbf{C}$. With $x=e^{2 \pi i \tau}, \vartheta_{1}$ satisfies

a)

$$
\begin{aligned}
& \vartheta_{1}(v \mid \tau)=2 x^{1 / 8} \sum_{m=0}^{\infty}(-1)^{m} \sin ((2 m+1) v) x^{m(m+1) / 2}, \\
&=i e^{\pi i[-v+(\tau / 4)]} \prod_{n=1}^{\infty}\left(1-x^{n}\right)\left(1-x^{(n-1)} e^{2 \pi i v}\right)\left(1-x^{n} e^{-2 \pi i v}\right), \\
& \vartheta_{1}(v+m \tau+n \mid \tau)=(-1)^{m+n} e^{-\pi i\left[2 m v+m^{2} \tau\right]} \vartheta_{1}(v \mid \tau) .
\end{aligned}
$$

c)

The Jacobi triple product identity is b); the other two follow from the definition.

For

$$
A=\left(\begin{array}{ll}
a & b \\
c & d
\end{array}\right) \in \Gamma(1) \text {, the transformation properties of } \eta(\tau) \text { and } \vartheta_{1}(v \mid \tau) \text { are: }
$$

$$
\eta\left(\frac{a \tau+b}{c \tau+d}\right)=v_{\eta}(A) \sqrt{c \tau+d} \eta(\tau)
$$

where

and

$$
v_{\eta}(A)= \begin{cases}\left(\frac{d}{c}\right)^{*} \exp \left(\frac{2 \pi i}{24}\left[-3 c-b d\left(c^{2}-1\right)+c(a+d)\right]\right), & \text { for } c \text { odd } \\ \left(\frac{c}{d}\right)_{*} \exp \left(\frac{2 \pi i}{24}\left[3 d-3-a c\left(d^{2}-1\right)+d(b-c)\right]\right), & \text { for } d \text { odd }\end{cases}
$$

$$
\vartheta_{1}\left(\frac{v}{c \tau+d} \mid \frac{a \tau+b}{c \tau+d}\right)=v_{\vartheta_{1}}(A) \sqrt{c \tau+d} e^{\pi i c v^{2} /(c \tau+d)} \vartheta_{1}(v \mid \tau)
$$

where

$$
v_{\vartheta_{1}}(A)=v_{\eta}^{3}(A)= \begin{cases}\left(\frac{d}{c}\right)^{*} \exp \left(\frac{2 \pi i}{8}[-3 c+c(a+d)]\right), & \text { for } c \text { odd } \\ \left(\frac{c}{d}\right)_{*} \exp \left(\frac{2 \pi i}{8}[3 d-3+d(b-c)]\right), & \text { for } d \text { odd }\end{cases}
$$

For (2.3b), (2.4), (2.5), and (2.6), see Rademacher [4, $\$ 74,80]$, although the specific form of $(2.5)$, using the extensions $\left(\frac{d}{c}\right)^{*}$ and $\left(\frac{c}{d}\right)_{*}$ of the Legendre symbols to allow the lower entry to be negative, follows Knopp [5]. (2.7) follows from (2.3b) by observing that

$$
\left.\frac{\partial}{\partial v} \vartheta_{1}(v \mid \tau)\right|_{v=0}=2 \pi \eta(\tau)^{3}
$$

Suppose $\rho, n \in \mathbf{Z}$ with $n \geq 1$ and $n \nmid \rho$. Then we define

$$
f_{n, \rho}(\tau)=-i(-1)^{[\rho / n \mathbf{l}} e^{\rho^{2}(\pi i / n) \tau} \vartheta_{1}(\rho \tau \mid n \tau),
$$

where 【 \denotes the greatest integer function. This has the product form, after the triple product $(2.3 \mathrm{c})$,

$$
f_{n, \rho}=c^{(n-2 \rho)^{2} / 8 n} \prod_{m=1}^{\infty}\left(1-x^{m n}\right)\left(1-x^{m n-\rho}\right)\left(1-x^{m n-(n-\rho)}\right) .
$$


LEMMA 2.1. If $A=\left(\begin{array}{ll}a & b \\ c & d\end{array}\right) \in \Gamma_{0}(n)$, then

$$
f_{n, \rho}(A \tau)=\left[(-1)^{\rho b+[\rho a / n]+[\rho / n]}\right]\left[e^{\rho^{2}(\pi i / n) a b} v_{\vartheta_{1}}\left({ }^{n} A\right)\right] \sqrt{c \tau+d} f_{n, \rho a}(\tau)
$$

Recall that ${ }^{n} A=\left(\begin{array}{cc}a & b n \\ c / n & d\end{array}\right)$, as in (1.3).

Proof. Using the transformation property (2.6) of $\vartheta_{1}$, we have

$$
\begin{aligned}
f_{n, \rho}(A \tau) & =(-1)^{[\rho / n]} e^{\rho^{2}(\pi i / n) A \tau} \vartheta_{1}\left(\frac{\rho(a \tau+b)}{c \tau+d} \mid \frac{a(n \tau)+n b}{\frac{c}{n}(n \tau)+d}\right) \\
& =(-1)^{[\rho / n]} v_{\vartheta_{1}}\left({ }^{n} A\right) \sqrt{c \tau+d} \exp \left(\rho^{2} \frac{\pi i}{n} A \tau\right) \exp \left(\pi i \frac{c}{n} \frac{(\rho(a \tau+b))^{2}}{c \tau+d}\right) \vartheta_{1}(\rho a \tau+\rho b \mid n \tau) \\
& =\left[(-1)^{[\rho / n]+\rho b} v_{\vartheta_{1}}\left({ }^{n} A\right)\right] \sqrt{c \tau+d} \exp \left(\rho^{2} \frac{\pi i}{n} A \tau(1+c(a \tau+b))\right) \vartheta_{1}(\rho a \tau \mid n \tau) \\
& =\left[(-1)^{[\rho / n]+\rho b} v_{\vartheta_{1}}\left({ }^{n} A\right)\right] \sqrt{c \tau+d} \exp \left(\rho^{2} \frac{\pi i}{n}\left(a^{2} \tau+a b\right)\right) \vartheta_{1}(\rho a \tau \mid n \tau) \\
& =\left[(-1)^{[\rho / n]+\rho b+[\rho a / n]}\right]\left[\exp \left(\rho^{2} \frac{\pi i}{n} a b\right) v_{\vartheta_{1}}\left({ }^{n} A\right)\right] \sqrt{c \tau+d} f_{n, \rho a}(\tau) .
\end{aligned}
$$

Since $f_{n, \rho+n}=f_{n,-\rho}=f_{n, \rho}$ and the quadratic reciprocity symbol satisfies $\left(\frac{r+5}{5}\right)=$ $\left(\frac{r}{5}\right)$ and $\left(\frac{-r}{5}\right)=\left(\frac{r}{5}\right)$, the following definition makes sense.

Definition 2.1. Suppose $\delta= \pm 1$. Then

$$
g_{\delta}(\tau)=\frac{f_{5, r}(\tau)}{\eta(\tau)},
$$

where $r$ is any integer with $\left(\frac{r}{5}\right)=\delta$.

We shall sometimes write $g_{+}$and $g_{-}$for $g_{+1}$ and $g_{-1}$. By the product expansion we see that

$$
\begin{aligned}
& g(\tau)=g_{-}(\tau)=x^{-1 / 60} G(x), \quad \text { and } \\
& h(\tau)=g_{+}(\tau)=x^{11 / 60} H(x) .
\end{aligned}
$$

DEFinition 2.2. For natural numbers $r$ and $s$ and for $\delta= \pm 1$, we define

$$
u_{\delta, r, s}=g_{-} \cdot g_{-\delta}+\delta g_{+} \cdot g_{\delta},
$$

If $r$ and $s$ satisfy $r s \equiv \pm 1(\bmod 5)$, then we define implicitly $\delta \in\{ \pm 1\}$ by $r+\delta s \equiv 0$ 
$(\bmod 5)$ and shall write

$$
u_{r, s}=u_{\delta, r, s}
$$

These modular forms are related to the power series $U_{r, s}$ of the introduction by:

We also define

$$
u_{r, s}(\tau)= \begin{cases}x^{-(r+s) / 60} U_{r, s}, & \text { when } r+s \equiv 0(\bmod 5), \\ x^{-(r-11 s) / 60} U_{r, s}, & \text { when } r-s \equiv 0(\bmod 5) .\end{cases}
$$

and

$$
p(\tau)=\frac{\eta(\tau)}{\eta(2 \tau)}=x^{-1 / 48} P(x)
$$

$$
p^{*}(\tau)=\frac{p(2 \tau)}{p(\tau)}=x^{-1 / 48} P^{*}(x) .
$$

THEOREM 2.4. $p(\tau)$ is a form of weight 0 on $\Gamma_{0}(2)$ with multiplier system

$$
v_{p}\left(\begin{array}{ll}
a & b \\
c & d
\end{array}\right)=\left(\frac{2}{d}\right) \exp \left(\frac{2 \pi i}{48}\left[-a c\left(d^{2}-1\right)-d(2 b+c)\right]\right),
$$

$p^{*}(\tau)$ is a modular form of weight 0 on $\Gamma_{0}(4)$ with multiplier system

$$
v_{p *}\left(\begin{array}{ll}
a & b \\
c & d
\end{array}\right)=\exp \left(\frac{2 \pi i}{96}\left[a c\left(d^{2}-1\right)-d(4 b-c)\right]\right),
$$

and $u_{r, s}$ is a modular form of weight 0 on $\Gamma_{0}([r, s])$ with multiplier system

$$
v_{r, s}=v_{1}\left|r . v_{1}\right| s \text {, }
$$

where $v_{1}(A)=v_{\eta}(A)^{14}$ denotes the multiplier system of $\eta^{14}(\tau)$, i.e.,

$$
v_{1}\left(\begin{array}{ll}
a & b \\
c & d
\end{array}\right)= \begin{cases}\exp \left(\frac{7 \pi i}{6}\left[-3 c-b d\left(c^{2}-1\right)+c(a+d)\right]\right), & c \text { odd } \\
\exp \left(\frac{7 \pi i}{6}\left[3 d-3-a c\left(d^{2}-1\right)+d(b-c)\right]\right), & d \text { odd }\end{cases}
$$

so then

$$
v_{r, s}\left(\begin{array}{ll}
a & b \\
c & d
\end{array}\right)= \begin{cases}\exp \left(\frac{7 \pi i}{6} \cdot \frac{r+s}{r s}\left[-3 c-b d\left(c^{2}-r s\right)+c(a+d)\right]\right), & c \text { odd } \\
\exp \left(\frac{7 \pi i}{6} \cdot \frac{r+s}{r s}\left[-a c\left(d^{2}-1\right)+d(r s b-c)\right]\right), & d \text { odd }\end{cases}
$$

Note that $v_{r, s}$ is the multiplier system of $\eta^{14}(r \tau) \cdot \eta^{14}(s \tau)$.

The assertions about $p(\tau)$ and $p^{*}(\tau)$ follow directly from the general property (1.4) about transforms $f \mid r$, and the transformation property (2.4) of $\eta(\tau)$. The rest of this section is devoted to proving the assertion for $u_{r, s}$. We shall first establish the 
transformation properties of $g_{\delta}(\tau)$, then use this to establish the multiplier system for $u_{r, s}$ on $\Gamma_{0}(5[r, s])$, and finally extend the result to $\Gamma_{0}([r, s])$.

In the transformations of $g_{\delta}$ we introduce a function $\chi_{5}(m)$ defined as follows:

$$
\chi_{5}(m)= \begin{cases}+1, & \text { for } m \equiv+1,+2(\bmod 5), \\ -1, & \text { for } m \equiv-1,-2(\bmod 5) .\end{cases}
$$

This function is determined by the property

$$
(-1)^{[m / 5]}=-\chi_{5}(m)\left(\frac{m}{5}\right)(-1)^{m}
$$

and satisfies

$$
\chi_{5}(m n)=(-1)^{\mathrm{neg}(m / 5) \operatorname{neg}(n / 5)} \chi_{5}(m) \chi_{5}(n),
$$

where neg $x=\left\{\begin{array}{ll}1, & \text { if } x<0, \\ 0, & \text { if } x \geq 0 .\end{array}\right.$ To see that $(2.20)$ is valid, let $m \equiv 2^{g}$, and $n \equiv 2^{h}(\bmod 5)$. Then $\chi_{5}(m)=(-1)^{g}$ and $(-1)^{(g+h)(g+h-1) / 2}=(-1)^{g(g-1) / 2+h(h-1) / 2+g h}$.

Proposition 2.5. For $A=\left(\begin{array}{ll}a & b \\ c & d\end{array}\right) \in \Gamma_{0}(5)$, we set $\delta^{\prime}=\left(\frac{a}{5}\right)$. Then the functions $g_{+}$and g- transform according to the rule

where

$$
g_{\delta}(A \tau)=v_{g, \delta}(A) \cdot g_{\delta \delta^{\prime}}(\tau)
$$

and

$$
v_{g, \delta}(A)=v_{0, \delta}(A) v_{1}(A)
$$

$$
v_{0, \delta}(A)=\chi_{5}(a)\left(\frac{a}{5}\right)^{\text {neg } \delta} e^{-\delta(4 \pi i / 5) a b}
$$

Thus, when $\left(\frac{a}{5}\right)=-1, g_{+}$transforms into $g_{-}$, and vice-versa.

Proof. We apply Lemma 2.1 , with $\rho$ chosen to be either 1 or 2 . Then, using $\frac{c}{5} \equiv 5 c(\bmod 24)$, and $\left(\frac{d}{c / 5}\right)=\left(\frac{a}{5}\right)\left(\frac{d}{c}\right)$, we get $v_{\vartheta_{1}}\left({ }^{5} A\right) / v_{\eta}(A)=\left(\frac{a}{5}\right) v_{1}(A)$. Then a couple of applications of (2.19), using the fact that $a b \equiv a+b+1(\bmod 2)$ for any $A \in \Gamma(1)$, and that $5 \rho+\rho^{2}=20-8 \delta$, gives us $(2.21)$.

COROllary. For any positive integer $r, g_{\delta}(r \tau)$ is a modular form of weight 0 on $\Gamma_{0,1}(5 r, 5)=\left\{A \in \Gamma_{0}(5 r) \mid a \equiv \pm 1(\bmod 5)\right\}$.

Proposition 2.6. Suppose $r, s$ are positive integers, $\delta \in\{ \pm\}$, and $r+\delta s \equiv 0(\bmod 5)$.

Then $u_{r, s}$ is a form of weight 0 on $\Gamma_{0}(5[r, s])$ with multiplier system $v_{r, s}(A)$. section.

We isolate the following sign argument for use here and again at the end of the 
Lemma. If $\delta, \delta^{\prime} \in\{ \pm 1\}$ and $R_{ \pm 1}, S_{ \pm 1}$ are any four quantities, then

$$
R_{-\delta^{\prime}} S_{-\delta \delta^{\prime}}+\delta R_{\delta^{\prime}} S_{\delta \delta^{\prime}}=\left(\delta^{\prime}\right)^{\text {neg } \delta}\left[R_{-1} S_{-\delta}+\delta R_{+1} S_{\sigma}\right]
$$

Proof. Check each of four cases.

Proof of Proposition 2.6. For any $m \in \mathbf{N}, \sigma \in\{ \pm 1\}, A=\left(\begin{array}{ll}a & b \\ c & d\end{array}\right) \in \Gamma_{0}(5 m)$, and $\delta^{\prime}=\left(\frac{a}{5}\right)$, we have

$$
g_{\sigma}(m A \tau)=v_{g, \delta}\left({ }^{m} A\right) g_{\sigma \delta^{\prime}}(m \tau),
$$

so for $A \in \Gamma_{0}(5[r, s])$, we have

$$
u_{r, s}(A \tau)=v_{g,-1}\left({ }^{r} A\right) v_{g,-\delta}\left({ }^{s} A\right)\left[g_{-\delta^{\prime}}(r \tau) g_{-\delta \delta^{\prime}}(s \tau)+\delta \xi g_{\delta^{\prime}}(r \tau) g_{\delta \delta^{\prime}}(s \tau)\right],
$$

where $\xi$ has the value

$$
\xi=\frac{v_{g,+}\left({ }^{r} A\right) v_{g, \delta}\left({ }^{s} A\right)}{v_{g,-}\left({ }^{r} A\right) v_{g,-\delta}\left({ }^{s} A\right)}=\left(\frac{a}{5}\right)^{-1-\delta} \exp 2\left(\frac{4 \pi i}{5} a b(r+\delta s)\right)=1,
$$

because $r+\delta s \equiv 0(\bmod 5)$. Applying the sign argument above, we have

$$
u_{r, s}(A \tau)=v_{g,-}\left({ }^{r} A\right) v_{g,-\delta}\left({ }^{s} A\right)\left(\frac{a}{5}\right)^{\text {neg } \delta} u_{r, s}(\tau) \text {. }
$$

Finally, applying the definition (2.23) of $v_{0, \delta}$.

$$
v_{g,-}\left({ }^{r} A\right) v_{g,-\delta}\left({ }^{s} A\right)\left(\frac{a}{5}\right)^{\mathrm{neg} \delta}=v_{0,-1}\left({ }^{r} A\right) v_{0,-\delta}\left({ }^{s} A\right)\left(\frac{a}{5}\right)^{\mathrm{neg} \delta} v_{1}\left({ }^{r} A\right) v_{1}\left({ }^{s} A\right)=v_{1}\left({ }^{r} A\right) v_{1}\left({ }^{s} A\right)
$$

and this completes the proof.

$A$ reduction in the proof of Theorem 2.4. Choose an integer $m$ such that

$$
\left\{\begin{array}{l}
m \equiv 0(\bmod 24 r s) \\
m \equiv 1(\bmod 5)
\end{array}\right.
$$

and define

$$
V=\left(\begin{array}{ll}
1 & 0 \\
m & 1
\end{array}\right)
$$

Then $\Gamma_{0}([r, s])$ is the group generated by $\Gamma_{0}(5[r, s])$ and $V$, and $v_{r, s}(V)=1$, so Theorem 2.4 follows if we know that

$$
u_{r, s}\left(\frac{\tau}{m \tau+1}\right)=u_{r, s}(\tau)
$$

We first prove two lemmas. The first gives $\vartheta_{1}\left(\frac{k}{5} \mid \frac{\tau}{5}\right)$ as a linear combination of $f_{5,1}$ and $f_{5,2}$. The other shows that $g_{\delta}\left(\frac{\tau}{m \tau+1}\right)$ is a certain linear combination of $g_{+}$and $g_{-}$. These two lemmas are then used to prove (2.28). 
Lemma 2.7. Suppose $k \in \mathbf{Z}, 5+k, \sigma=\left(\frac{k}{5}\right)$, and $\omega=\frac{\sqrt{5}+1}{2}$. Then

$$
\vartheta_{1}\left(\frac{k}{5} \mid \frac{\tau}{5}\right)=-i 5^{1 / 4} \chi_{5}(k)(-1)^{k}\left[\sigma \omega^{-\sigma / 2} f_{5,2}(\tau)-\omega^{\sigma / 2} f_{5,1}(\tau)\right]
$$

Proof. Choose $\rho \in\{1,2\}$ so that $k \equiv \pm \rho(\bmod 5)$. Then $\left(\frac{\rho}{5}\right)=\left(\frac{k}{5}\right)=\sigma$ and

$$
\vartheta_{1}\left(\frac{k}{5} \mid \frac{\tau}{5}\right)=(-1)^{[k / 5]} \vartheta_{1}\left(\frac{\rho}{5} \mid \frac{\tau}{5}\right)=-\chi_{5}(k) \sigma(-1)^{k} \vartheta_{1}\left(\frac{\rho}{5} \mid \frac{\tau}{5}\right)
$$

so it suffices to show that

$$
\vartheta_{1}\left(\frac{\rho}{5} \mid \frac{\tau}{5}\right)=5^{1 / 4}\left[\omega^{-\sigma / 2} f_{5,2}(\tau)-\sigma \omega^{\sigma / 2} f_{5,1}(\tau)\right]
$$

$\begin{aligned} & \text { Setting } s_{m}= \frac{\sin \left(\frac{(2 m+1) \pi \rho}{5}\right)}{\sin \left(\frac{\pi \rho}{5}\right)} \text {, and using the series expansion (2.3a) } \\ & i \vartheta_{1}\left(\frac{\rho}{5} \mid \frac{\tau}{5}\right)=2 i \sin \frac{\pi \rho}{5} \sum_{m=0}^{\infty}(-1)^{m} s_{m} \exp \pi i \frac{\tau}{5}\left(m+\frac{1}{2}\right)^{2} .\end{aligned}$

Since $\cos \frac{\pi}{5}=\frac{\sqrt{5}+1}{4}=\frac{1}{2} \omega$, we have

$$
2 \sin \left(\frac{\pi \rho}{5}\right)=5^{1 / 4} \omega^{-\sigma / 2}, \quad \text { and } \quad 2 \cos \left(\frac{\pi \rho}{5}\right)=\omega^{\sigma}
$$

Setting $c=2 \cos \frac{\pi \rho}{5}$ we get

$$
s_{m+1}=c s_{m}-s_{m-1} \text {. }
$$

Then, by induction, $s_{5 m}=1, s_{5 m+1}=\sigma \omega^{\sigma}, s_{5 m+2}=0, s_{5 m+3}=-\sigma \omega^{\sigma}$, and $s_{5 m+4}=-1$, for all $m$. Putting these into the above series and collecting together the terms with $m \equiv 0$ and $m \equiv 4(\bmod 5)$ and likewise the terms with $m \equiv 1$ and $m \equiv 3(\bmod 5)$, and rearranging the series, we obtain

$$
i \vartheta_{1}\left(\frac{\rho}{5} \mid \frac{\tau}{5}\right)=5^{1 / 4} \omega^{-\sigma / 2}\left[e^{4(\pi i / 5) \tau} \vartheta_{1}(2 \tau \mid 5 \tau)-\sigma \omega^{\sigma} e^{(\pi i / 5) \tau} \vartheta_{1}(\tau \mid 5 \tau)\right]
$$

which is $(2.30)$, and the lemma is proven.

LemMa 2.8. Suppose $\delta^{\prime} \in\{ \pm 1\}, 8 \mid k$ and $5 \nmid k$. Let $\sigma=\left(\frac{k}{5}\right)$ and $\delta^{\prime \prime}=\delta^{\prime} \sigma$. We define

$$
R_{\delta^{\prime \prime}}(k)=\left[\left(\delta^{\prime \prime} \omega^{\delta^{\prime \prime} / 2} e^{-(4 \pi i / 5) \sigma k}\right) \cdot g_{-}(\tau)-\left(\omega^{-\delta^{\prime \prime} / 2} e^{-(4 \pi i / 5) \sigma k}\right) \cdot g_{+}(\tau)\right],
$$


and

Then

$$
\xi_{\delta^{\prime}}(k)=i \delta^{\prime} \chi_{5}(k) \sigma^{\left(1+\delta^{\prime}\right) / 2} e^{-\delta^{\prime}(4 \pi i / 5) \sigma k}
$$

$$
g_{\delta} \cdot\left(\frac{\tau}{k \tau+1}\right)=5^{-1 / 4} \xi_{\delta^{\prime}}(k) R_{\delta^{\prime \prime}}(k) .
$$

Proof. We shall do the proof only for the case $\delta^{\prime}=-1$, the case $\delta^{\prime}=+1$ being analogous. In this case we have $\delta^{\prime \prime}=-\sigma$, and $g_{\delta^{\prime}}(\tau)=g(\tau)=e^{(4 \pi i / 5) \tau} \vartheta_{1}(2 \tau \mid 5 \tau) / \eta(\tau)$.

Let $\beta=-\sigma k=-\left(\frac{k}{5}\right) k$. Then $\beta k \equiv-1(\bmod 5)$, so with $\alpha=\frac{\beta k+1}{5}$, we have $\left(\begin{array}{ll}5 & \beta \\ k & \alpha\end{array}\right) \in \Gamma(1)$ and

$$
\left(\begin{array}{ll}
5 & 0 \\
0 & 1
\end{array}\right)\left(\begin{array}{ll}
1 & 0 \\
k & 1
\end{array}\right)=\left(\begin{array}{ll}
5 & \beta \\
k & \alpha
\end{array}\right)\left(\begin{array}{cc}
1 & -\beta \\
0 & 5
\end{array}\right)
$$

Setting $z=\frac{\tau-\beta}{5}$, we have

$$
5 \cdot \frac{\tau}{k \tau+1}=\frac{5 z+\beta}{k z+\alpha}, \text { and } 2 \cdot \frac{\tau}{k \tau+1}=\frac{2 \tau / 5}{k z+\alpha} .
$$

By the transformation properties (2.4)-(2.7) of $\eta(\tau)$ and $\vartheta_{1}(v \mid \tau)$, we get

$$
g\left(\frac{\tau}{k \tau+1}\right)=\exp \left(\left(4 \frac{\pi i}{5}\right)\left(\frac{\tau}{k \tau+1}\right)\right) \cdot \frac{v_{\vartheta_{1}}\left(\begin{array}{ll}
5 & \beta \\
k & \alpha
\end{array}\right)}{v_{\eta}\left(\begin{array}{ll}
1 & 0 \\
m & 1
\end{array}\right)} \frac{1}{\sqrt{5}} \exp \left(\pi i k \frac{(2 \tau / 5)^{2}}{(k \tau+1) / 5}\right) \cdot \frac{\vartheta_{1}\left(\frac{2 \tau}{5} \mid z\right)}{\eta(\tau)}
$$

Since $5 \alpha-\beta k=1$ and $\beta \equiv k \equiv 0(\bmod 24)$, we have $\left(\frac{k}{\alpha}\right)_{*}=\left(\frac{k}{5}\right)\left(\frac{k}{5 \alpha}\right)_{*}=\left(\frac{k}{1-\sigma k^{2}}\right)_{*}\left(\frac{k}{5}\right)=$ $\sigma$ and $\alpha \equiv 5(\bmod 8)$, so we get $v_{\vartheta_{1}}\left(\begin{array}{ll}5 & \beta \\ k & \alpha\end{array}\right)=\sigma$ and also $v_{\eta}\left(\begin{array}{ll}1 & 0 \\ k & 1\end{array}\right)=1$. Hence (2.34) becomes

$$
\begin{aligned}
\eta(\tau) g\left(\frac{\tau}{k \tau+1}\right) & =\frac{\sigma}{\sqrt{5}} e^{4(\pi i / 5) \tau} \vartheta_{1}\left(\frac{2 \tau}{5} \mid \frac{\tau-\beta}{5}\right) \\
& =5^{-1 / 2} \sigma e^{-(4 \pi i / 5) \beta} \vartheta_{1}\left(\frac{2 \beta}{5} \mid \frac{\tau-\beta}{5}\right) \\
& =-5^{-1 / 2} e^{(4 \pi i / 5) \sigma k} \vartheta_{1}\left(\frac{2 k}{5} \mid \frac{\tau+\sigma k}{5}\right) \\
& =i 5^{-1 / 4} \chi_{5}(2 k) e^{(4 \pi i / 5) \sigma k}\left[-\sigma \omega^{\sigma / 2} f_{5,2}(\tau+\sigma k)-\omega^{-\delta / 2} f_{5,1}(\tau+\sigma k)\right]
\end{aligned}
$$

Now, since $\delta^{\prime \prime}=\delta^{\prime} \sigma=-\sigma$, we get (2.33) by applying the transformations property of $f_{5, \rho}$ from Lemma 2.1, and the definition (2.9) of $g_{ \pm}$. 
Conclusion of the proof of (2.2) and Theorem 2.4. Let $\sigma=\left(\frac{r}{5}\right)$. Then from $r+\delta s \equiv 0(\bmod 5)$ and $m \equiv 1(\bmod 5)$, it follows that $\left(\frac{r}{5}\right)=\left(\frac{m / r}{5}\right)=\left(\frac{m / s}{5}\right)=\sigma$, and
$r^{2} \equiv s^{2} \equiv \sigma(\bmod 5)$ and $r s \equiv-\delta \sigma(\bmod 5)$. Hence

and

$$
\chi_{5}\left(\frac{m}{r}\right)=\chi_{5}\left(\sigma r^{2} \frac{m}{r}\right)=\chi_{5}(\sigma m r)=\sigma \chi_{5}(r)
$$

$$
\chi_{5}\left(\frac{m}{r}\right) \chi_{5}\left(\frac{m}{s}\right)=\chi_{5}(r) \chi_{5}(s)=(-1)^{\mathrm{neg} \sigma} \chi_{5}(r s)=\sigma \chi_{5}(-\delta \sigma)=-\delta .
$$

For $\delta^{\prime} \in\{ \pm 1\}$ we define

$$
R_{\delta^{\prime}}=\left[\delta^{\prime} \omega^{-\delta^{\prime} / 2} e^{(4 \pi i / 5) \sigma(m / r)} g_{-}(r \tau)-\omega^{\delta^{\prime} / 2} e^{-(4 \pi i / 5) \sigma(m / f)} g_{+}(r \tau)\right]
$$

Then the last lemma, with $\delta^{\prime}=\sigma \delta=\left(\frac{m / r}{5}\right) \delta$, says

Similarly, with

$$
g_{\delta}\left(r \frac{\tau}{m \tau+1}\right)=g_{\delta}\left(\frac{r \tau}{\frac{m}{r}(r \tau)+1}\right)=5^{-1 / 4} \xi_{\delta}\left(\frac{m}{r}\right) R_{\sigma \delta}
$$

$$
S_{\delta^{\prime}}=\left[\delta^{\prime} \omega^{-\delta^{\prime} / 2} e^{(4 \pi i / 5) \sigma(m / s)} g_{-}(s \tau)-\omega^{\delta^{\prime} / 2} e^{-(4 \pi i / 5) \sigma(m / s)} g_{+}(s \tau)\right],
$$

we have

\section{Setting}

$$
g_{\delta}\left(s \frac{\tau}{m \tau+1}\right)=5^{-1 / 4} \xi_{\delta}\left(\frac{m}{s}\right) S_{\sigma \delta}
$$

we then have

$$
\xi_{+}=\xi_{+}\left(\frac{m}{r}\right) \xi_{\delta}\left(\frac{m}{s}\right) \quad \text { and } \quad \zeta_{-}=\xi_{-}\left(\frac{m}{r}\right) \xi_{-\delta}\left(\frac{m}{r}\right)
$$

$$
u_{r, s}\left(\frac{\tau}{m \tau+1}\right)=5^{-1 / 2}\left[\zeta_{-} R_{-\sigma} S_{-\sigma \delta}+\delta \xi_{+} R_{\sigma} S_{\sigma \delta}\right]
$$

We use the definition (2.32) of $\xi_{-}$and $\xi_{+}$, and the values (2.35) and (2.36) of $\chi_{5}$ to calculate

$$
\begin{aligned}
\zeta_{-} & =\left[i(-1) \chi_{5}\left(\frac{m}{r}\right) \exp \left(\frac{4 \pi i}{5} \sigma \frac{m}{r}\right)\right] \times\left[i(-\delta) \chi_{5}\left(\frac{m}{s}\right) \sigma^{\mathrm{neg} \delta} \exp \left(\delta \frac{4 \pi i}{5} \sigma \frac{m}{s}\right)\right] \\
& =-\delta \chi_{5}\left(\frac{m}{r}\right) \chi_{5}\left(\frac{m}{s}\right) \sigma^{\mathrm{neg} \delta} \exp \left(\frac{4 \pi i}{5} \sigma \frac{m}{r s}(r+\delta s)\right) \\
& =-\delta(-\delta) \sigma^{\mathrm{neg} \delta} \\
& =\sigma^{\mathrm{neg} \delta}
\end{aligned}
$$


and, similarly,

so then

$$
\zeta_{+}=\sigma^{\mathrm{neg} \delta}
$$

$$
\begin{aligned}
u_{r, s}\left(\frac{\tau}{m \tau+1}\right) & =\sigma^{\mathrm{neg} \delta}\left[R_{-\sigma} S_{-\sigma \delta}+\delta R_{\sigma} S_{\sigma \delta}\right] \cdot 5^{-1 / 2} \\
& =\left[R_{-} S_{-\delta}+\delta R_{+} S_{\delta}\right] \cdot 5^{-1 / 2},
\end{aligned}
$$

by the sign argument (2.24).

For $\delta=+1$, since $\frac{m}{r}+\frac{m}{s}=0(\bmod 5)$, and $\omega+\frac{1}{\omega}=\sqrt{5}$, we have

$$
\begin{aligned}
u_{r, s}\left(\frac{\tau}{m \tau+1}\right)= & 5^{-1 / 2}\left[g_{-}(r \tau) g_{-}(s \tau) \exp \left(\frac{4 \pi i}{5} \sigma\left(\frac{m}{r}+\frac{m}{s}\right)\left(\omega+\frac{1}{\omega}\right)\right)\right. \\
& \left.+g_{+}(r \tau) g_{+}(s \tau) \exp \left(\frac{4 \pi i}{5} \sigma\left(\frac{m}{r}+\frac{m}{s}\right)\left(\frac{1}{\omega}+\omega\right)\right)\right] \\
= & u_{r, s}(\tau) .
\end{aligned}
$$

This proves (2.28) and completes the proof of Theorem 2.4.

\section{Orders of the functions.}

LEMMA 3.1. Suppose $a, b, c, d$ are real, $c \neq 0$, and suppose $f(\tau)=\vartheta_{1}(a \tau+b \mid c \tau+d)$ does not vanish identically. Then when $f$ is expanded as a power series in $x=e^{2 \pi i r}$, the lowest order term has degree

$$
\frac{c}{2}\left[\left(\frac{a}{c}-\llbracket \frac{a}{c} \rrbracket-\frac{1}{2}\right)^{2}-\frac{a^{2}}{c^{2}}\right] .
$$

Proof. From (2.6) we get $f(\tau)=\sum_{-\infty}^{\infty} \xi_{n} x^{\alpha_{n}}$, where $\alpha_{n}$ is a quadratic expression in $n$ whose minimum for integral values of $n$ is the expression in (3.1). The coefficient of this term vanishes only when $\frac{a}{c} \in \mathbf{Z}$ and $\frac{a d-b c}{c} \in \mathbf{Z}$. But then, from $(2.3 \mathrm{a}, \mathrm{b})$, we would have $f(\tau) \equiv 0$.

Lemma 3.2. If $(a, c)=1, n \nmid \rho$ and $e=(n, c)$, then

$$
\operatorname{ord}\left(f_{n, \rho} ; \frac{a}{c}\right)=\frac{e^{2}}{2 n}\left(\frac{a \rho}{e}-\llbracket \frac{a \rho}{e} \rrbracket-\frac{1}{2}\right)^{2} \text {. }
$$

Proof. Choose $b, d \in \mathbf{Z}$ so that $A=\left(\begin{array}{ll}a & b \\ c & d\end{array}\right) \in \Gamma(1)$. Since $A \infty=\frac{a}{c}$, we have $\operatorname{ord}\left(f ; \frac{a}{c}\right)=\operatorname{ord}(f \mid A ; \infty)$. Then

$$
f_{n, \rho}(A \tau)=(-1)^{[\rho / n]} e^{\rho^{2}(\pi i / n) A \tau} \vartheta_{1}\left(\frac{\rho(a \tau+b)}{c \tau+d} \mid \frac{n a \tau+n b}{c \tau+d}\right)
$$


Let $e=(n, c)$ and $m=\frac{n}{e}$. Then there is $k$ so that $\delta=\frac{d e-c k}{n} \in \mathbf{Z}$. Finally let $\beta=b e-a k$ and $B=\left(\begin{array}{ll}m a & \beta \\ c / e & \delta\end{array}\right)$. Then $B \in \Gamma(1)$, and

$$
\frac{n a \tau+n b}{c \tau+d}=\frac{e(a \tau+b)}{\frac{1}{m}(c \tau+d)}=\frac{a m\left(\frac{e \tau+k}{m}\right)+\beta}{\frac{c}{e}\left(\frac{e \tau+k}{m}\right)+\delta},
$$

so that, after applying (2.5) and doing some simplifications as in the proof of Lemma 2.1,

$$
f_{n, \rho}(A \tau)=\sqrt{\frac{c \tau+\bar{d}}{m}} \xi \exp \left(\frac{\rho^{2}}{n} \pi i a^{2} \tau\right) \vartheta_{1}\left(\frac{\rho a}{m} \tau+\frac{\rho b}{m} \mid \frac{e}{m} \tau+\frac{k}{m}\right),
$$

for an appropriate constant $\xi$. Hence, by Lemma 3.1,

$$
\begin{aligned}
\operatorname{ord}\left(f_{n, p} ; \frac{a}{c}\right) & =\frac{(\rho a)^{2}}{2 n}+\frac{e}{2 m}\left[\left(\frac{a \rho}{e}-\llbracket \frac{a \rho}{e} \rrbracket-\frac{1}{2}\right)^{2}-\left(\frac{a \rho}{e}\right)^{2}\right] \\
& =\frac{e^{2}}{2 n}\left(\frac{a \rho}{e}-\llbracket \frac{a \rho}{e} \rrbracket-\frac{1}{2}\right) .
\end{aligned}
$$

THEOREM 3.3. Suppose $\rho \in\{1,2\}, \delta=\left(\frac{\rho}{5}\right)$, and $a$, c are integers with $(a, c)=1$. Then

$$
\operatorname{ord}\left(g_{\delta} ; \frac{a}{c}\right)=\left\{\begin{aligned}
\frac{11}{16}, & \text { when }\left(\begin{array}{l}
a \\
c
\end{array}\right) \equiv \pm\left(\begin{array}{l}
\rho \\
0
\end{array}\right)(\bmod 5) \\
-\frac{1}{60}, & \text { otherwise. }
\end{aligned}\right.
$$

4. Applying the Fricke involutions. The modulary group of level $n$ is the set $G_{n}$ of $2 \times 2$ matrices whose entries are residue classes modulo $n$ with determinant $1(\bmod n)$.

LEMMA 4.1. The mapping $\Gamma(1) \rightarrow G_{n}$ is onto.

The map referred to is, of course, reading the entries modulo $n$. For a proof see Schoeneberg [9].

We shall add additional conditions to those of the definition (0.9) of the Fricke involution in order to simplify the multiplier systems that will arise. We assume that $M$ is given by $M=\left(\begin{array}{ll}a & b \\ c & d\end{array}\right)\left(\begin{array}{cc}m & 0 \\ 0 & 1\end{array}\right)$ with

and

$$
a b \equiv a c \equiv c d \equiv b c \equiv 0\left(\bmod 2^{\alpha} 3^{\beta} 5^{\gamma}\right)
$$

$$
a \equiv d \equiv 1(\bmod 5), \quad \text { if } 5 \nmid m,
$$


where $\alpha, \beta, \gamma$ are sufficiently large natural numbers. Usually, sufficiently large will mean $\alpha \geq 3, \beta \geq 1$, and $\gamma \geq 1$. We can also require, when it helps,

$$
\begin{cases}d \equiv 1(\bmod 8), & \text { when } m \text { is odd, and } \\ c \equiv 1(\bmod 8), & \text { when } m \text { is even. }\end{cases}
$$

The consistency of (4.1), (4.2), and (4.3) is guaranteed by the lemma.

LEMMA 4.2. If $A=\left(\begin{array}{ll}a & b \\ c & d\end{array}\right) \in \Gamma(1) \quad$ and $a b \equiv a c \equiv b d \equiv c d \equiv 0(\bmod 120)$, then

$$
\begin{aligned}
& v_{\eta}(A)= \begin{cases}\left(\frac{d}{c}\right)^{*} i^{-c / 2}, & \text { c odd }, \\
\left(\frac{c}{d}\right) i_{*}(d-1) / 2 & \text { d odd },\end{cases} \\
& v_{1}(A)= \begin{cases}\left(\frac{a}{5}\right) i^{c}, & \text { c odd }, \\
\left(\frac{a}{5}\right) i^{1-d}, & d \text { odd },\end{cases} \\
& v_{g, \delta}=\chi_{5}(a)\left(\frac{a}{5}\right)^{(1-\delta) / 2} \times \begin{cases}i^{c}, & \text { c odd }, \\
i^{1-d}, & d \text { odd } .\end{cases}
\end{aligned}
$$

Proof. If $c$ is odd, then $c^{2} \equiv 1(\bmod 8)$ and we need only use $b d \equiv 0(\bmod 3)$ and $c(a+d) \equiv 0(\bmod 24)$ and $(1.2)$ to ensure that $v_{\eta}(A)=\left(\frac{d}{c}\right)^{*} e^{(2 \pi i / 8)(-3 c)}=\left(\frac{d}{c}\right)^{*} i^{-c / 2}$. The rest follow similarly from (1.2), (1.17), and (1.18).

The conditions $a \equiv d \equiv 1(\bmod 5)$ and $c \equiv 1$ or $d \equiv 1(\bmod 8)$ were not considered here because they are not preserved under the transition $A \mapsto_{r} A$.

Proposition 4.3. Suppose $r|n, s| n$, and $m \| n$ and let the matrix $M=$ $A\left(\begin{array}{cc}m & 0 \\ 0 & 1\end{array}\right)$ with $A=\left(\begin{array}{ll}a & b \\ c & d\end{array}\right) \in \Gamma(1)$, with $m \mid d$, and $\frac{n}{m} \mid c$ be a Fricke involution of level $m$ for $\Gamma_{0}(n)$, chosen so that (4.1) and (4.2) hold. Suppose $\delta \in\{ \pm 1\}$ so that $r+\delta s \equiv 0(\bmod 5)$.

Then

$$
u_{r, s} \mid M=\xi u_{r^{\prime}, s^{\prime}},
$$

where $\xi, r^{\prime}$, and $s^{\prime}$ are determined by

$$
\begin{array}{lll}
r_{1}=(r, m), & r_{2}=\frac{r}{(r, m)}, & r^{\prime}=\frac{r m}{(r, m)^{2}}, \\
s_{1}=(s, m), & s_{2}=\frac{s}{(s, m)}, & s^{\prime}=\frac{s m}{(s, m)^{2}},
\end{array}
$$


and

$$
\xi=\left(\frac{r_{1}}{5}\right)^{(1+\delta) / 2}\left(\frac{s_{1}}{5}\right)^{(1-\delta) / 2} \chi_{5}\left(r_{1} s_{1}\right) \times \begin{cases}(-1)^{(1 / 2)\left(r_{2}+s_{2}\right)}, & \text { m even }, \\ (-1)^{(1 / 2)\left(r_{1}+s_{2}\right)}, & \text { modd }\end{cases}
$$

If, in addition, $[m, 2 r] \mid n$, and if $p(\tau)=\frac{\eta(\tau)}{\eta(2 \tau)}$, then

$$
p|r| M= \begin{cases}\left(\frac{2}{r_{1}}\right) p \mid r^{\prime}, & \text { modd, } \\ \left(\frac{2}{r_{2}}\right) \frac{\sqrt{2}}{p \mid \frac{r^{\prime}}{2}}, & \text { m even. }\end{cases}
$$

Proof. For $r \in \mathbf{N}, \delta \in\{ \pm 1\}$, we have

$$
g_{\delta}|r| M=g_{\delta}\left|\left(\begin{array}{cc}
r & 0 \\
0 & 1
\end{array}\right) A\right| m=g_{\delta}\left|A^{\prime}\left(\begin{array}{cc}
r_{2} & 0 \\
0 & r_{1}
\end{array}\right)\right| m=v_{g, \delta}\left(A^{\prime}\right) g_{\delta \delta^{\prime}} \mid r^{\prime},
$$

where $\delta=\left(\frac{r_{1} a}{5}\right)$ and $A^{\prime}={ }_{r_{2}}^{r_{1}} A=\left(\begin{array}{cc}a r_{1} & b r_{2} \\ c / r_{2} & d / r_{1}\end{array}\right)$. Applying the congruences (4.1) and (4.2), and proposition 2.5 , we have

$$
v_{g, \delta}\left(A^{\prime}\right)=\chi_{5}\left(r_{1}\right)\left(\frac{r_{1}}{5}\right)^{(1-\delta) / 2} \times \begin{cases}i^{r_{2}}, & m \text { even } \\ i^{1-r_{1}}, & m \text { odd }\end{cases}
$$

We have used the fact that when $m$ is even, $r_{2}$ is odd, so $\frac{c}{r_{2}} \equiv r_{2} c \equiv r_{2}(\bmod 4)$, and, similarly, $\frac{d}{r_{1}} \equiv r_{1}(\bmod 4)$ when $m$ is odd. Consequently,

where

$$
\begin{aligned}
u_{r, s} \mid M & =\xi_{0}\left(g_{\delta^{\prime}}\left|r^{\prime} \cdot g_{\delta \delta^{\prime \prime}}\right| s^{\prime}+\delta \xi_{1} g_{-\delta^{\prime}}\left|r^{\prime} \cdot g_{-\delta \delta^{\prime \prime}}\right| s^{\prime}\right) \\
& =\xi_{0} \xi^{\mathrm{neg} \delta^{\prime}} \cdot u_{r^{\prime}, s^{\prime}},
\end{aligned}
$$

$$
\xi_{0}=\chi_{5}\left(r_{1}\right) \chi_{5}\left(s_{1}\right)\left(\frac{s_{1}}{5}\right)^{(1-\delta) / 2} \times \begin{cases}i^{r_{2}+s_{2}}, & m \text { even } \\ i^{2-r_{1}-s_{1}}, & m \text { odd }\end{cases}
$$

and

$$
\delta^{\prime}=\left(\frac{r_{1}}{5}\right), \quad \delta^{\prime \prime}=\left(\frac{s_{1}}{5}\right), \quad \xi=\delta \delta^{\prime} \delta^{\prime \prime}
$$

Since $\delta^{\text {neg } \delta^{\prime}}=\delta^{\prime \text { neg } \delta}$, and $\delta^{\prime \text { neg } \delta^{\prime}}=\delta^{\prime}$, we have

$$
\begin{aligned}
& \xi_{0} \xi^{\text {neg } \delta^{\prime}}=\chi_{5}\left(r_{1}\right) \chi_{5}\left(s_{1}\right) \delta^{\prime \prime(1-\delta) / 2} \delta^{\prime(1-\delta) / 2} \delta^{\prime} \delta^{\prime \prime\left(1-\delta^{\prime}\right) / 2}, \\
& =\chi_{5}\left(r_{1} s_{1}\right)\left(\frac{r_{1}}{5}\right)^{(1+\delta) / 2}\left(\frac{s_{1}}{5}\right)^{(1-\delta) / 2} \times \begin{cases}(-1)^{(1 / 2)\left[\left(r / r_{1}\right)+\left(s / s_{1}\right)\right]}, & m \text { even, } \\
-(-1)^{(1 / 2)\left(r_{1}+s_{1}\right)}, & m \text { odd, }\end{cases}
\end{aligned}
$$

when $m \mid r s$. 
For $p(\tau)$, we have, when $m$ is odd,

where

$$
p|r| M=2^{1 / 4} \frac{\eta|r| M}{\eta|2 r| M}=2^{1 / 4} \frac{v_{\eta}\left({ }_{r_{1}} A\right) \eta \mid r^{\prime}}{v_{\eta}\left({ }_{r_{1}}^{2 r_{2}} A\right) \eta \mid 2 r^{\prime}}=\xi \cdot p \mid r^{\prime},
$$

$$
\xi=\frac{\left(\frac{c / r_{2}}{d / r_{1}}\right)_{*} i^{-\left(d / r_{1}-1\right) / 2}}{\left(\frac{c / 2 r_{2}}{d / r_{1}}\right)_{*} i^{-\left(d / r_{1}-1\right) / 2}}=\left(\frac{2}{r_{1}}\right),
$$

and similarly, when $m$ is even,

$$
p|r| M=2^{1 / 4} \frac{v_{\eta}\left({ }_{r_{1}}^{r_{2}} A\right) \eta \mid r^{\prime}}{v_{\eta}\left(\begin{array}{c}
r_{2} \\
2 r_{1}
\end{array}\right) \eta \mid \frac{r^{\prime}}{2}}=\left(\frac{2}{r_{2}}\right) \frac{\sqrt{2}}{p \mid \frac{r^{\prime}}{2}} .
$$

5. Proving the identities. We now use the properties derived above to prove the identities stated in $\$ 0$. This is mostly a matter of some calculation. We do the identity (\#16) in some detail and give only a few intermediate results of the calculation in the rest.

The first step is to calculate the multiplier systems of each term in the identity. Second we compute the result of applying the Fricke involutions and observe what effect this has on the orders at the cusps. If there are any cusps which are not obtained from $\infty$ by the application of one of the Fricke involutions, we use the formula (1.5) for the (invariant) order of a transform to get a lower bound for the order at the remaining cusps. Finally we calculate the necessary number of terms in the series expansion to prove the identity from the valence formula (0.4).

(\#16). We set $Q(x)=P(x) P\left(x^{7}\right)$, and $Q^{*}(x)=P^{*}(x) P^{*}\left(x^{7}\right)$, and

$$
I D(x)=U_{6,14}-\frac{1}{2 x}\left[Q^{*}(x) Q\left(x^{3}\right)-Q(x) Q^{*}\left(x^{3}\right)\right]
$$

and obtain from it a modular form

$$
I(\tau)=x^{-1 / 3} I D(x)=u_{6,14}-\frac{1}{2}\left[q^{*} \cdot q\left|3-q \cdot q^{*}\right| 3\right],
$$

where $q(\tau)=p(\tau) p(7 \tau)$, and $q^{*}(\tau)=p^{*}(\tau) p^{*}(7 \tau)$. Each factor of each term of $I$ is a form of weight 0 on $\Gamma_{0}(84), q$ and $q^{*}$ each have multiplier system

$$
v_{q}(A)=\exp \left(\frac{2 \pi i}{3} d\left(-b+\frac{c}{28}\right),\right)
$$

(see (4.8)) and $q(3 \tau)$ and $q^{*}(3 \tau)$ have the multiplier system

$$
v_{q} \mid 3(A)=v_{q}\left({ }^{3} A\right)=\exp \left(\frac{2 \pi i}{3} d\left(-3 b+\frac{c}{84}\right)\right)
$$


so $q^{*} \cdot q \mid 3$ and $q \cdot q^{*} \mid 3$ have multiplier system

$$
v_{q} \cdot v_{q} \mid 3=\exp \left(\frac{2 \pi i}{3} d\left(-b+\frac{c}{84}\right)\right)
$$

which agrees with the multiplier system $v_{6,14}$ of $u_{6,14}$ (cf. (4.5)), so $I$ is a modular form of weight 0 on $\Gamma_{0}(84)$.

We compute the effect of the Fricke involutions $M_{3}, M_{4}, M_{7}$, the rest being obtained by $M_{12}=M_{3} M_{4}, M_{21}=M_{3} M_{7}, M_{28}=M_{4} M_{7}$, and $M_{84}=M_{3} M_{4} M_{7}$. (Cf. Proposition 4.3.) The result is

$$
\begin{array}{cc}
u_{6,14}\left|M_{3}=u_{6,14}\right| M_{4}=-u_{6,14} \mid M_{7}=u_{6,14}, \\
\left(q^{*} \cdot q \mid 3\right)\left|M_{3}=q \cdot q^{*}\right| 3, & \left(q \cdot q^{*} \mid 3\right)\left|M_{3}=q^{*} \cdot q\right| 3, \\
\left(q^{*} \cdot q \mid 3\right)\left|M_{7}=-q^{*} \cdot q\right| 3, & \left(q \cdot q^{*} \mid 3\right)\left|M_{7}=-q\right| 3 \cdot q^{*}, \\
\left(q^{*} \cdot q \mid 3\right) \mid M_{4}=-2 \frac{q^{*}}{q \mid 3}, & \left(q \cdot q^{*} \mid 3\right) \mid M_{4}=-2 \frac{q^{*} \mid 3}{q \mid 2}
\end{array}
$$

Hence, setting $I_{d}=I \mid M_{d}$, we get

and

$$
I=I_{3}=-I_{7}=-I_{21},
$$

$$
I_{4}=I_{12}=-I_{28}=-I_{84}=-u_{6,14}+\left(\frac{q^{*}}{q \mid 6}-\frac{q^{*} \mid 3}{q \mid 2}\right) .
$$

We obtain a power series $I D_{4}(x)$ from $I_{4}$ by $I_{4}=x^{-1 / 3} I D_{4}(x)$. We do not need to know precisely the form of $\mathrm{ID}_{4}(x)$, as we will see below, we only need to know that it does not involve any negative powers of $x$, which it does not.

Suppose now that we calculate $m_{1}$ terms of $I D(x)$, i.e., the coefficients of $1, x, x^{2}, \ldots, x^{m_{1}-1}$, and $m_{4}$ terms of $I D_{4}(x)$ and these all turn out to be 0 . Then we know

But

$$
\operatorname{Ord}_{\Gamma_{0}(84)}(I ; \infty) \geq m_{1}-\frac{1}{3}, \quad \operatorname{Ord}_{\Gamma_{0}(84)}\left(I_{4} ; \infty\right) \geq m_{4}-\frac{1}{3} \text {. }
$$

$$
\operatorname{Ord}_{\Gamma_{0}(84)}\left(I ; \frac{1}{d}\right)=\operatorname{Ord}_{\Gamma_{0}(84)}\left(l_{d} ; \infty\right)
$$

so we know a lower bound for $\operatorname{Ord}_{\Gamma_{0}(84)}\left(I ; \frac{1}{d}\right)$ for $d=1,3,4,7,12,21,28$, and 84 . The function $I$ has no poles in the upper half-plane, so we have $\operatorname{Ord}_{\Gamma_{0}(84)}(I ; z) \geq 0$ for all $z \in \mathbf{H}$. We still need a lower bound for the orders at $\frac{1}{d}$ for $d=2,6,14,42$. To get these, we need only calculate a bound for $d=2$, because, for $d$ odd,

$$
\operatorname{Ord}_{\Gamma_{0}(84)}\left(I ; \frac{1}{2 d}\right)=\operatorname{Ord}_{\Gamma_{0}(84)}\left(I_{d} ; \frac{1}{2}\right)=\operatorname{Ord}_{\Gamma_{0}(84)}\left(I ; \frac{1}{2}\right) \text {. }
$$

We have, by the definition of the invariant order,

$$
\operatorname{Ord}_{\Gamma_{0}(84)}\left(I ; \frac{1}{2}\right)=21 . \operatorname{ord}\left(1 ; \frac{1}{2}\right),
$$


and we apply the transformation property (1.5) of the invariant order, together with the orders for $g=g_{-}$, and $h=g_{+}$from Theorem 3.1 to get

so that

$$
\begin{aligned}
& \operatorname{ord}\left(g \mid 6 ; \frac{1}{2}\right)=-\frac{1}{90}, \quad \operatorname{ord}\left(g \mid 14 ; \frac{1}{2}\right)=-\frac{1}{210}, \\
& \operatorname{ord}\left(h \mid 6 ; \frac{1}{2}\right)=-\frac{1}{90}, \quad \operatorname{ord}\left(h \mid 14 ; \frac{1}{2}\right)=-\frac{1}{210},
\end{aligned}
$$

Also,

$$
\operatorname{ord}\left(u_{6,14} ; \frac{1}{2}\right) \geq-\frac{1}{63} \text {. }
$$

$$
\begin{aligned}
& \operatorname{ord}\left(q ; \frac{1}{2}\right)=-\frac{1}{21}, \quad \operatorname{ord}\left(q \mid 3 ; \frac{1}{2}\right)=-\frac{1}{63}, \\
& \operatorname{ord}\left(q^{*} ; \frac{1}{2}\right)=\frac{2}{21}, \quad \text { and } \quad \operatorname{ord}\left(q^{*} ; \frac{1}{2}\right)=\frac{2}{63}
\end{aligned}
$$

follow from ord $\left(\eta(\tau) ; \frac{r}{s}\right)=\frac{1}{24}$ for all $\frac{r}{s} \in \mathbf{Q}^{*}$, together with the definitions of $q$ and $q^{*}$. Thus

$$
\operatorname{Ord}_{\Gamma_{0}(84)}\left(I ; \frac{1}{2}\right) \geq 21 . \min \left\{-\frac{1}{63}, \frac{5}{63}, \frac{5}{63}\right\}=-\frac{1}{3} .
$$

Combining the above bounds gives us

$$
\sum_{z \in \mathscr{F}^{*}} \operatorname{Ord}_{\Gamma_{0}(84)}(I ; z) \geq 4\left(m_{1}-\frac{1}{3}\right)+4\left(m_{4}-\frac{1}{3}\right)+4\left(-\frac{1}{3}\right)=4\left(m_{1}+m_{4}-1\right) .
$$

The identity will be proven, i.e., $I \equiv 0$, if

$$
m_{1}+m_{4}>1 \text {. }
$$

However, we can easily calculate in the ring of formal power series modulo the ideal $\left\langle x^{2}\right\rangle$ :

and

$$
U_{6,14} \equiv 1 \text {, }
$$

$$
\frac{1}{2 x}\left[P^{*}(x) P\left(x^{3}\right) P^{*}\left(x^{7}\right) P\left(x^{21}\right)-P(x) P^{*}\left(x^{3}\right) P\left(x^{7}\right) P^{*}\left(x^{21}\right)\right] \equiv \frac{1}{2 x}[(1+x)-(1-x)]=1,
$$

so $m_{1} \geq 2$, and (\#16) is proven.

For the remaining identities we give only the modular form variation $I$ of the identity, the multiplier system $v(A)$ for $I$, the results $I_{d}=I \mid M_{d}$ of applications of the Fricke involutions, and the inequality for the valence formula, assuming that $m$ terms of the power series have been calculated. None of the rest have cusps which are not the result of $\infty$ after application of the involutions, so that part of the calculation can be omitted.

(\#15).

$$
\begin{gathered}
I=x^{-1 / 6} I D(x)=u_{3,7}-u_{21,1} . \\
v(A)=v_{3,7}=v_{21,1} . \\
I=-I_{3}=-I_{6}=I_{21} . \\
\sum_{z \in \mathscr{F}^{*}} \operatorname{Ord}_{\Gamma_{0}(21)}(I ; z) \geq 4\left(m-\frac{1}{6}\right) .
\end{gathered}
$$


(\#18). For the computation of $I_{2}$ and $I_{13}$ here we need the identity (\#17) from the complete list of forty, which was proven by Bressoud [2]. It is

$$
u_{2,13}=u_{26,1} \text {. }
$$

We could prove this here with virtually the same computations as for (\#15). We take it as known.

We set $q(\tau)=p(13 \tau) / p(\tau)$.

$$
\begin{gathered}
I=x^{-1 / 2} I D(x)=u_{2,13}^{2}-q+\frac{1}{q}, \\
v(A)=(-1)^{b-(c / 26)} . \\
I=I_{2}=I_{13}=I_{26} . \\
\sum_{z \in \mathscr{F}^{*}} \operatorname{Ord}_{\Gamma_{0}(26)}(I ; z) \geq 4\left(m-\frac{1}{2}\right) .
\end{gathered}
$$

(\#34). We set $u=u_{2,23} \cdot u_{46,1}$ and $q(\tau)=p(\tau) p(23 \tau)$.

$$
\begin{gathered}
I=x^{-1} I D(x)=u-q-1-\frac{2}{q} . \\
v(A)=1 . \\
I=I_{2}=I_{23}=I_{46} . \\
\sum_{z \in \mathscr{F}^{*}} \operatorname{Ord}_{\Gamma_{0}(46)}(I ; z) \geq 4(m-1) .
\end{gathered}
$$

(\#36) and (\#37).

$$
\begin{gathered}
I=x^{-25 / 24} I D(x)=p(11 \tau) u_{2,33}-p(3 \tau) u_{66,1} . \\
I^{\prime}=x^{-11 / 24} I D^{\prime}(x)=p(33 \tau) u_{11,6}-p(\tau) u_{22,3} . \\
v(A)=v^{\prime}(A)=1 . \\
I=I_{33}, \quad I_{66}=-I_{2}=\frac{-\sqrt{2}}{p(11 \tau) p(3 \tau)} I \\
I^{\prime}=I_{3}=I_{11}, \quad I_{6}=I_{22}=\frac{\sqrt{2}}{p(\tau) p(33 \tau)} I^{\prime} . \\
\sum_{z \in \mathscr{F}^{*}} \operatorname{Ord}_{\Gamma_{0}(66)}(I ; z) \geq 4\left(m-\frac{25}{24}\right)+2\left(\frac{14}{24}\right)+4\left(m^{\prime}-\frac{11}{24}\right)+2\left(\frac{34}{24}\right)=4\left(m+m^{\prime}-\frac{1}{2}\right) .
\end{gathered}
$$

(\#40). We set $u=u_{1,94} u_{47,2}$, and $q(\tau)=p(\tau) p(47 \tau)$.

$$
\begin{gathered}
I=x^{-4} I D(x)=\left[u-q-2-\frac{2}{q}\right]^{2}-\left[4 q+9+\frac{8}{q}\right] . \\
v(A)=1 . \\
I=I_{2}=I_{47}=I_{94} \\
\sum_{z \in \mathscr{F}^{*}} \operatorname{Ord}_{\Gamma_{0}(94)}(I ; z) \geq 4(m-4) .
\end{gathered}
$$


It is rather remarkable that very few terms need to be computed any of these series.

The remaining identity. The only identity of the forty not proven after this is

where

$$
U_{19,4} P\left(x^{38}\right)=U_{76,1}^{*} P\left(x^{2}\right),
$$

$$
U_{76,1}^{*}=G\left(x^{76}\right) H^{*}(x)+x^{15} G^{*}(x) H\left(x^{76}\right),
$$

and $H^{*}(x)=H(-x), G^{*}(x)=G(-x)$. From the infinite products defining $H(x)$ and $G(x)$, we obtain

So we define

$$
H^{*}(x)=H(x)\left[\frac{G\left(x^{2}\right)}{H(x)}\right]^{2} \frac{H\left(x^{2}\right)}{G\left(x^{4}\right)}, \quad \text { and } \quad G^{*}(x)=G(x)\left[\frac{H\left(x^{2}\right)}{G(x)}\right]^{2} \frac{G\left(x^{2}\right)}{H\left(x^{4}\right)} .
$$

so that

$$
g_{\delta}^{*}(\tau)=g_{\delta}(\tau)\left[\frac{g_{-\delta}(2 \tau)}{g_{\delta}(\tau)}\right]^{2} \frac{g_{\delta}(2 \tau)}{g_{-\delta}(4 \tau)}
$$

and we let

$$
g_{+}^{*}(\tau)=x^{11 / 60} H^{*}(x) \text { and } g_{-}^{*}(\tau)=x^{-1 / 60} G^{*}(x) \text {, }
$$

$$
u_{76,1}^{*}(\tau)=g(76 \tau) h^{*}(\tau)+h(76 \tau) g^{*}(\tau) .
$$
From (1.2) and (2.21), it follows that for $A=\left(\begin{array}{ll}a & b \\ c & d\end{array}\right) \in \Gamma_{0}(20), g_{\delta}^{*}(\tau)$ transforms according
to

$$
g_{\delta}^{*}(A \tau)=v_{g, \delta}^{*}(A) \cdot g_{\delta \delta}^{*}(\tau),
$$

where $\delta^{\prime}=\left(\frac{a}{5}\right)$, and

$$
v_{g, \delta}^{*}=\chi_{5}(a)\left(\frac{a}{5}\right)^{\operatorname{pos}(\delta)} \exp \delta \frac{4 \pi i}{5} a b \exp \left(\frac{\pi i}{6}\left[3 d-3-a c\left(d^{2}-1\right)+d\left(-5 b+\frac{c}{4}\right)\right]\right)
$$

From this we get $u_{76,1}^{*}$ is a form on $\Gamma_{0}(380)$ with multiplier system

$$
v_{76,1}^{*}(A)=\exp \left(\frac{\pi i}{6}\left[a c\left(d^{2}-1\right)+d(-b+c)\right],\right)
$$

as we did for $u_{r, s}$ in Proposition 2.6. Then we check that both sides of (\#35) have the same multiplier system, which they do.

It appears that a further reduction, as was possible for $u_{r, s}$ in Theorem 2.4 , to $\Gamma_{0}(76)$ may not be possible; the identity (\#24) in Birch's list specifically lacks this final symmetry and it involves a similar combination, i.e. $u_{1,1}^{*}=g(\tau) h^{*}(\tau)-h(\tau) g^{*}(\tau)$.

At any rate, the apparent lack of symmetry for the factor 5 forces somewhat more calculation. We let $M_{d}$, for $d=4,19,76$ be Fricke involutions for $\Gamma_{0}(380)$ and calculate:

$$
\begin{aligned}
& I_{1}=p(2 \tau) \cdot u_{76,1}^{*}-p(38 \tau) \cdot u_{19,4}, \\
& I \mid M_{19}=\sim I_{19}=p(38 \tau) \cdot u_{4,19}^{*}-p(2 \tau) \cdot u_{76,1}, \\
& I \mid M_{4}=\sim I_{4}=\frac{u_{19,1}^{*}}{p(\tau)}-\frac{u_{76,1}}{p(19 \tau)}, \\
& I \mid M_{76}=\sim I_{76}=\frac{u_{1,19}^{*}}{p(19 \tau)}-\frac{u_{19,4}}{p(\tau)},
\end{aligned}
$$


where

$$
\begin{aligned}
& u_{19,1}^{*}(\tau)=g(19 \tau) g^{*}(\tau)-h(19 \tau) h^{*}(\tau) \\
& u_{1,19}^{*}(\tau)=g(\tau) g^{*}(19 \tau)-h(\tau) h^{*}(19 \tau), \\
& u_{4,19}^{*}(\tau)=h(4 \tau) g^{*}(19 \tau)+g(4 \tau) h^{*}(19 \tau) .
\end{aligned}
$$

We can use our knowledge of the orders of $g, h$, and $p$ to get a lower bound

$$
\operatorname{Ord}_{\Gamma_{0}(380)}\left(I_{d} ; \frac{1}{r}\right) \geq m_{d, r}
$$

for $d=1,4,19,76$ at any of the cusps $\frac{1}{r}, r \mid 380$ of $\Gamma_{0}(380)$. We can also calculate some number $n_{d}$ of terms in the series $I D_{d}(x)$ associated with $I_{d}$. Then since

$$
I_{1}=x^{-7 / 6} I D_{1}, \quad I_{4}=x^{-9 / 8} I D_{4}, \quad I_{19}=x^{-7 / 24} I D_{19}, \quad \text { and } \quad I_{76}=x^{+11 / 24} I D_{76},
$$

we would have

$$
\begin{aligned}
\sum_{r \mid 380} \operatorname{Ord}_{\Gamma_{0}(380)}\left(I ; \frac{1}{r}\right) \geq\left(n_{1}-\frac{7}{6}\right)+\left(n_{4}-\frac{7}{24}\right)+\left(n_{19}-\frac{9}{8}\right)+\left(n_{76}+\frac{11}{24}\right)+ \\
m_{1,10}+m_{19,10}+m_{1,1}+m_{4,1}+m_{19,1}+m_{76,1}+m_{1,2}+m_{19,2} .
\end{aligned}
$$

(These estimates correspond to the cusps $\infty, \frac{1}{20}, \frac{1}{95}, \frac{1}{5}, \frac{1}{10}, \frac{1}{190}, \frac{1}{1}, \frac{1}{4}, \frac{1}{19}, \frac{1}{76}, \frac{1}{2}$, and $\frac{1}{38}$.) The resulting values for the $m_{d, r}$ are, respectively,

$$
-\frac{59}{120},-\frac{41}{120},-\frac{41}{24},-\frac{118}{12},-\frac{59}{24},-\frac{172}{12},-\frac{59}{24},-\frac{41}{120} \text {. }
$$

This gives us a lower bound of

$$
\sum_{r \mid 380} \operatorname{Ord}_{\Gamma_{0}(380)}\left(I ; \frac{1}{r}\right) \geq n_{1}+n_{4}+n_{19}+n_{76}-\frac{291}{8}>\sum n_{d}-37 .
$$

Hence, if the first 10 terms of each of the four identities vanish, then the valence formula tells us that $I=0$. They do, so the proof of (\#35) is complete.

\section{REFERENCES}

1. B. J. Birch, A look back at Ramanujan's manuscripts, Math. Proc. Camb. Phil. Soc., 78 (1975), 73-79.

2. David Bressoud, Proof and generalization of certain identities conjectured by Ramanujan (thesis, Temple university 1980).

3. H. B. C. Darling, Proofs of certain identities and congruences enunciated by $S$. Ramanujan, Proc. London Math. Soc. (2), 19 (1921), 350-372. 387-397.

4. L. J. Rogers, On a type of modular relation, Proc. London Math. Soc. (2), 19 (1921),

5. L. J. Mordell, Note on certain modular relations considered by Messrs. Ramanujan, Darling and Rogers, Proc. London Math. Soc. (2), 20 (1922), 408-416.

6. G. N. Watson, Proof of certain identities in combinatory analysis, J. Indian Math. Soc., 20 (1933), 57-69. 
7. Robert Rankin, Modular forms and functions, (Cambridge University Press 1977).

8. Bruno Schoeneberg, Elliptic modular functions, (Springer-Verlag 1974).

9. Marvin I. Knopp, Modular functions in analytic number theory, (Markham 1970).

10. Hans Rademacher, Topics in analytic number theory, (Springer-Verlag 1972).

11. Bruce C. Berndt, Anthony J. F. Biagioli, and James M. Purtilo, Ramanujan's modular equations of "large" prime degree, Journal of the Indian Math. Soc. 51 (1987).

12. Bruce C. Berndt, Anthony J. F. Biagioli, and James M. Purtilo, Ramanujan's modular equations of degrees 7 and 11, Indian Journal of Mathematics 29 (1987).

13. Bruce C. Berndt, Anthony J. F. Biagioli, and James M. Purtilo, Ramanujan's "mixed" modular equations, J. Ramanujan Math. Soc. 1 (1 \& 2) 1986, 1-25.

The University of Missouri at Rolla,

Rolla

Missouri 65401

U.S.A. 